\title{
Evaluation of the Effect of Nursing Intervention on the Curative Effect and Living Ability of Elderly Patients with Myocardial Infarction
}

\author{
Junying $\mathrm{Gao}^{1, \mathrm{a}}$ \\ ${ }^{1}$ Henan Province Hospital of Traditional Chinese Medicine Zhengzhou, Henan 450000
}

\begin{abstract}
This article is to explore the effect of applying nursing intervention methods on the life ability of elderly patients with myocardial infarction. The method of this reserch is to take patients in our hospital as an example to carry out research work. The study selected patients who were treated in our hospital from December 2019 to December 2020 as an example. The researchers selected all elderly patients with myocardial infarction, and the number of patients was selected as 100 cases. The different nursing methods of patients were divided into two groups --- the conventional nursing methods and comprehensive nursing methods were applied respectively, named the control group and the research group, and the nursing effects of the two groups of patients were compared and analyzed. The effective rates of the two groups of patients were $98.00 \%$ and $82.00 \%$ respectively. At admission, the patients' BNP, living ability and psychological scores were significantly higher than those of the control group. There was a big difference in data between the groups, $P>0.05$. After nursing intervention, the patients in the research group had better scores compared with the control group, $\mathrm{P}<0.05$, which was statistically significant, and the research group had better nursing effects. The application of comprehensive nursing methods in elderly patients with myocardial infarction has a significant clinical effect, which can improve the living ability of the patients and is of positive significance for the recovery of the patients.
\end{abstract}

\section{Introduction}

As far as acute myocardial infarction is concerned, it mainly refers to the situation that the coronary blood supply drops sharply or is interrupted in the case of coronary artery disease, which leads to myocardial ischemic necrosis. The patients are mostly elderly. After the onset, it is usually accompanied by arrhythmia and heart failure and other adverse conditions, which have a negative impact on the life, health and quality of life of the patient ${ }^{[1]}$. In this case, promoting the recovery of patients with acute myocardial infarction and improving the quality of life of patients is an important content in clinical development ${ }^{[2]}$. Based on this, the research group will apply comprehensive nursing methods to explore its effects on patients.

\section{Objective}

A comparative experiment was carried out, taking elderly patients with myocardial infarction as an example, applying comprehensive nursing and conventional nursing methods, and comparing the effects of the two methods on the life ability of patients.

\section{Methods}

\subsection{General information of patients}

The selected research site is our hospital, and the research period is from December 2019 to December 2020. The patient selection criteria are elderly patients with myocardial infarction, and the number is 100 . The patients were first divided into two groups with 50 people in each group, and given patients nursing intervention. The methods were conventional nursing and comprehensive nursing methods. The groups were named the control group and the research group. In the reseach group, the oldest patient was 87 years old and the youngest 53 years old, and the average age of the patients was $(67.56 \pm 4.34)$ years. In the control group, the maximum and minimum ages of patients were 88 and 52 years old, respectively, and the average age of patients was $(66.98 \pm 5.04)$ years. The general data of the two groups of children were compared, and the results indicated that $\mathrm{P}>0.05$, which was comparable.

Inclusion criteria: All patients were diagnosed with acute myocardial infarction, and the patients developed ischemic chest pain within 12 hours of onset; the patients were informed of this research work and signed an

\footnotetext{
agaojunying20010407@163.com
} 
informed consent form; this research work was approved by the hospital ethics committee.

Exclusion criteria: incomplete data of patient; impaired consciousness; patient has a history of mental illness; patient has poor compliance and withdrew from the study halfway.

\subsection{Research methods}

The patients in the control group applied traditional nursing methods. After the patients were admitted to the hospital, they were given ECG monitoring, the patients' heart rate, blood pressure and blood oxygen saturation were monitored, and the patients were given health education on myocardial infarction diseases, and health guidance was given to the patients.

Patients in the research group apply comprehensive nursing methods. In the specific implementation process, the patients are first given health education. After the patients are admitted to the hospital, they will be actively communicated to discuss the pathogenesis, treatment methods and prognosis of myocardial infarction. Inform patients of effects and related precautions, increase the patient's attention, ensure that patients correctly recognize their own diseases, reduce unnecessary worry, and ensure that patients actively cooperate with treatment. Secondly, give patients environmental care, introduce the hospital environment to patients, help patients adapt as soon as possible, reduce their negative psychology, and effectively control indoor temperature and humidity, regularly carry out ward cleaning and disinfection, ensure the indoor environment, and open windows regularly for ventilation. Arrange some indoor potted plants and books, and play music appropriately to ensure that patients are in a relaxed state. At the same time, provide patients with psychological care, analyze patient's psychological state, develop active communication with patients, guide patients to communicate, and provide psychological counseling to patients until patient's bad psychological problem is cleared, ease patient's mood, and ensure that patients is at the best state to receive treatment. Meanwhile, medication care is given to patients. For elderly patients with myocardial infarction, they need to take related medications for a long time. Nursing staff need to pay more attention to instruct patients to take medications on time, and explain the active ingredients of drugs to patients. Applicable symptoms and contraindications, etc., to improve patient awareness and ensure patients compliance with medication. Finally, give patients rehabilitation care, actively encourage patients to get out of bed 3 days after the operation is completed, and promote patients to get out of bed as soon as possible. The activity time is 15 minutes each time, twice a day, and then increase the time and frequency of activities every day. Based on patients' tolerability, to ensure that patients recover as soon as possible.

\subsection{Observation indicators}

Effect evaluation: It mainly includes three indicators, namely significant, effective and ineffective. Significant mainly refers to the disappearance of the patient's angina pectoris symptoms and the return of the ECG examination results to normal. Effective means that the patient's angina pectoris symptoms have been relieved and under the ECG examination, the heterozygous ST segment decreased, but it did not return to the normal level. Ineffective means that patient's various clinical symptoms have not been significantly improved. The total effective rate of this study is to exclude inefficiency.

BNP determination: The immunofluorescence method is applied to determine the BNP value of patients before treatment and after continuous treatment.

Ability of daily living: The ability of daily living scale is applied to measure the patient's living ability when the patient is admitted and discharged. The items include a total of 14 items and a total score of 56 points. A score of 14 is for normal function of patients and a score of 14 or more means that the ability of daily living is reduced to varying degrees, the higher the score, the poorer living ability of the patients ${ }^{[3]}$.

Psychological score: compare the psychological scores of patients before and after treatment, apply SAS and SDS self-rating scale, SDS score of 53 means depression, SAS score of 50 or more means anxiety, the higher the score, the more serious the depression and anxiety ${ }^{[4]}$.

\subsection{Statistical methods}

Use the statistical software SPSS20.0 as a tool to perform statistical analysis on the data appearing in this study, verify the comparison results of the measurement data $(\mathrm{x} \pm \mathrm{s})$ with the $t$ value, and compare the count data (n, \%) The results were verified by the $\boldsymbol{X}^{2}$ value. When the result showed $\mathrm{P}<0.05$, it indicated that the difference between the groups had statistical analysis value.

\section{Results}

\subsection{Therapeutic effect}

The effective rates of the two groups of patients were $98.00 \%$ and $82.00 \%$, respectively, and the difference in data was statistically significant $(\mathrm{P}<0.05)$.

Table1. Comparison of effective treatment rates between the two groups of patients $[\mathrm{n}(\%)]$

\begin{tabular}{|c|c|c|c|c|}
\hline Group & Significant & Effective & Ineffective & $\begin{array}{c}\text { Effective } \\
\text { rate }\end{array}$ \\
\hline $\begin{array}{c}\text { Research } \\
\text { group } \\
(\mathbf{n}=\mathbf{5 0})\end{array}$ & $\begin{array}{c}37 \\
(74.00)\end{array}$ & $\begin{array}{c}12 \\
(24.00)\end{array}$ & $1(2.00)$ & $\begin{array}{c}49 \\
(98.00)\end{array}$ \\
\hline $\begin{array}{l}\text { Control } \\
\text { group } \\
(n=50)\end{array}$ & $\begin{array}{c}23 \\
(46.00)\end{array}$ & $\begin{array}{c}18 \\
(36.00)\end{array}$ & $\begin{array}{c}9 \\
(18.00)\end{array}$ & $\begin{array}{c}41 \\
(82.00)\end{array}$ \\
\hline$X^{2}$ & & & & 9.004 \\
\hline $\mathrm{P}$ & & & & $<0.05$ \\
\hline
\end{tabular}




\subsection{BNP determination}

At admission, the BNP scores of the two groups of patients were relatively high, with no significant difference, $\mathrm{P}>0.05$. When the patients were discharged, their scores were all reduced, and the scores of the research group were lower than those of the control group. The difference was significant at $\mathrm{P}<0.05$.

Table2. Comparison of BNP determination $(\mathrm{x} \pm \mathrm{s})$

\begin{tabular}{ccc}
\hline Group & Admission & Discharged \\
\hline $\begin{array}{c}\text { Research group } \\
(\mathbf{n = 5 0}) \\
\begin{array}{c}\text { Control group } \\
(\mathbf{n}=\mathbf{5 0})\end{array} \\
\mathbf{t}\end{array}$ & $148.56 \pm 62.12$ & $58.09 \pm 19.08$ \\
$\mathbf{P}$ & 0.985 & 8.998 \\
\hline
\end{tabular}

\subsection{Living ability measurement}

The difference between the two groups of patients' admission living ability measurement scores was small, $\mathrm{P}>0.05$. After discharge, their daily living ability scores were improved. In contrast, patients in the research group scored higher, with large data differences, $\mathrm{P}<0.05$.

Table3. Determination of the living ability of the two groups of patients $(\mathrm{x} \pm \mathrm{s})$

\begin{tabular}{ccc}
\hline Group & Admission & Discharged \\
\hline $\begin{array}{c}\text { Research group } \\
(\mathbf{n}=\mathbf{5 0}) \\
\begin{array}{c}\text { Control group } \\
(\mathbf{n}=\mathbf{5 0})\end{array}\end{array}$ & $57.05 \pm 3.76$ & $18.05 \pm 2.11$ \\
$\mathrm{t}$ & $57.24 \pm 4.09$ & $35.45 \pm 3.23$ \\
$\mathrm{P}$ & 0.554 & 5.445 \\
& $>0.05$ & $<0.05$ \\
\hline
\end{tabular}

\subsection{Psychological score}

Before nursing, the psychological scores of the two groups of patients were similar, $\mathrm{P}>0.05$. After nursing intervention, the scores changed. The SAS score and SDS score of the research group were lower than those of the control group, $\mathrm{P}<0.05$, there was a difference.

Table4. Comparison of psychological scores between the two groups $(\mathrm{x} \pm \mathrm{s})$

\begin{tabular}{llccc}
\hline \multirow{2}{*}{ Group } & \multicolumn{2}{c}{ SAS } & \multicolumn{2}{c}{ SDS } \\
\cline { 2 - 5 } & $\begin{array}{c}\text { Before } \\
\text { nursing }\end{array}$ & $\begin{array}{c}\text { After } \\
\text { nursing }\end{array}$ & $\begin{array}{c}\text { Before } \\
\text { nursing }\end{array}$ & $\begin{array}{c}\text { After } \\
\text { nursing }\end{array}$ \\
\hline
\end{tabular}

\begin{tabular}{ccccc}
\hline $\begin{array}{c}\text { Research } \\
\text { group } \\
(\mathbf{n = 5 0})\end{array}$ & $66.98 \pm 6.09$ & $47.09 \pm 4.53$ & $58.09 \pm 6.74$ & $42.09 \pm 4.66$ \\
$\begin{array}{c}\text { Control } \\
\text { group } \\
(\mathbf{n}=\mathbf{5 0})\end{array}$ & $67.09 \pm 7.03$ & $51.09 \pm 5.00$ & $58.09 \pm 6.42$ & $51.23 \pm 5.09$ \\
$\mathrm{t}$ & 7.887 & 10.097 & 0.865 & \\
& & & & \\
$\mathrm{P}$ & $>0.05$ & $<0.05$ & $>0.05$ & $<0.05$ \\
\hline
\end{tabular}

\section{Conclusion}

The clinical morbidity of acute myocardial infarction is relatively high. After the onset of the patient, there is usually greater psychological pressure, which leads to arrhythmia in the patient, and the incidence of sudden death is relatively high ${ }^{[5]}$. According to the results of relevant research data, elderly patients with acute myocardial infarction are prone to negative psychological problems, which have a greater impact on the quality of life and ability of the patients ${ }^{[6]}$. It is necessary to increase clinical attention to this problem and apply comprehensive nursing methods in elderly patients with acute myocardial infarction. The comprehensive nursing method is based on the patient-centered, providing comprehensive and systematic nursing services for patients, ensuring that nursing staff will strengthen all aspects of nursing work, improving patient self-confidence in treatment, actively cooperating with various nursing work, and providing patients with a comfortable ward environment, to popularize disease knowledge for patients, and to give patients psychological counseling, so as to ensure that patients receive treatment in the best state, improve nursing effects, and promote patient recovery ${ }^{[7]}$. After the application of comprehensive nursing methods, the results showed that patients in the research group had higher effective rates, better psychological scores, lower BNP scores, and stronger living abilities. All the data are significantly different from the control group $(\mathrm{P}<0.05)$, indicating that the comprehensive nursing method has a significant clinical application effect.

In summary, the application of comprehensive nursing methods in elderly patients with myocardial infarction has significant significance. It can improve clinical nursing effects, ensure nursing quality, and improve patients' unhealthy emotions, and can be promoted and used in clinical practice.

\section{Acknowledgement}

I would like to express my sincere gratitude to the people who provided help to this paper. The successful completion of this paper is all thanks to the help of these people. Thank you for your guidance on my thesis, which helped continuous improvement of the thesis and selfgrowth. I would like to express my heartfelt blessings and sincere thanks. 


\section{Reference}

1. Li Haiyan, Shen Xiangqiao. Analysis of the impact of collaborative nursing intervention on the disease cognition of elderly patients with myocardial infarction[J]. International Journal of Nursing, 2019, 38(12):1849-1851.

2. Wu Juandong. The effect of nursing intervention on depression and quality of life in elderly patients with myocardial infarction[J]. Electronic Journal of Integrated Traditional Chinese and Western Medicine Cardiovascular Diseases, 2020, 008(009): 155-156.

3. Zhu Hongdan. The value of early rehabilitation nursing intervention on the cardiac function and short-term prognosis of elderly patients with myocardial infarction and arrhythmia[J]. Health for All, 2019, No.502(17):199-200.

4. Wang Wei, Zhao Qiaoyan. The effect of high-quality nursing intervention on the prognosis of patients with myocardial infarction[J]. Modern Medicine and Health, 2020.

5. Ma Xiaorong, Zhang Yu. The effect of high-quality nursing intervention on the rehabilitation effect of elderly patients with acute myocardial infarction after percutaneous coronary intervention[J]. Clinical Medical Research and Practice, 2019, 004(029):177179.

6. Zhang Chong. Study on the effect of personalized nursing intervention on ventricular function and ability of daily living in acute myocardial infarction[J]. Chinese Medical Guide, 2020, 018(005):218-219.

7. Gao Jingjing. Observation on the effect of nursing intervention on depression and quality of life in elderly patients with myocardial infarction[J]. World Latest Medical Information Abstracts (Continuous Electronic Journal) 2020, Volume 20, Issue 86, 361$362,2020$. 